\title{
A Simple, Fast and Highly Sensitive Colorimetric Detection of Zein in Aqueous Ethanol via Zein-Pyridine-Gold Interactions
}

\author{
Alejandro Latorre-Sanchez ${ }^{a, b}$ and Jose A. Pomposo ${ }^{* a, b, c}$

\footnotetext{
a. Centro de Física de Materiales (CSIC, UPV/EHU) - Materials Physics Center, Paseo Manuel de Lardizabal 5, E-20018 San Sebastián, Spain. Email: Josetxo.pomposo@ehu.eus

b. Departamento de Física de Materiales, Universidad del País Vasco (UPV/EHU), Apartado 1072, E-20800 San Sebastián, Spain

c. IKERBASQUE - Basque Foundation for Science, María Díaz de Haro 3, E-48013 Bilbao, Spain
}

\section{Formation of gold nanoparticles in aqueous ethanol in the presence of pyridine- functionalized single-chain nanoparticles allows for the fast and highly sensitive colorimetric detection of zein corn protein.}

Zein is the main prolamine protein in corn.1 Prolamins are major storage proteins that contain nitrogen for seed germination. Because zein is insoluble in water, resistant to grease and microbial attack, it has found traditional uses in coatings, plastics, textiles and adhesives, as well as recent new uses by taking advantage of its biological properties for supporting growing cells, delivering drugs and producing both degradable sutures and biodegradable plastics.2-6 As a result of its particular amino acid sequence and structure, zein is insoluble in water but soluble in aqueous ethanol which has become a reference solvent for this hydrophobic protein. It has been estimated that more than half of the solvent-accessible surface of zein is occupied by hydrophobic residues.7

In recent years, the enhanced demand of renewable materials has increased the interest in more efficient zein extraction procedures. Zein is normally produced from a protein-rich coproduct called corn gluten meal obtained by corn wet-milling. 8 The major component of commercial zein is Zzin, having two subtypes, Z19 and Z22, named according to their respective molecular weights (in $\mathrm{kDa}$ ). As more corn is being used in the so-called dry-grind ethanol process, there is more interest in zein extraction from this process. 8 In addition to the development of higher efficient and selective zein extraction procedures; new analytic methods allowing the simple, fast and highly sensitive colorimetric detection of zein in aqueous ethanol are certainly required. In particular, a previously reported colorimetric method based on stained zein spots on chromatography paper9 was found to be sensitive for zein concentrations $>100 \quad$ 1.ghtten using this method, more than $1 \mathrm{~h}$ of work-up per sample, including staining, destaining, elution and UV absorbance reading at $595 \mathrm{~nm}$ is required.

Herein, we develop a simple, fast, highly sensitive and robust colorimetric detection of zein in aqueous ethanol based on the formation of gold nanoparticles (AuNPs) in the presence of pyridine-functionalized single-chain nanoparticles (ScNPs). ScNps are versatile soft nano-objects synthesized by compaction of individual polymer 
chains through single-chain technology.10-13 In recent years, several colorimetric sensors have been developed based on AuNPs due to their unique optical properties, long-term stability and friendly biocompatibility with biomacromolecules.14 The use of ScNPs as sensors of metal ions has been reported, 15 as well as the synthesis of AuNPs using ScNPs as nanoreactors.16 However, to our best knowledge, the synergistic sensing capabilities offered by combining AuNPs and functional ScNPs have not been exploited for the rapid and efficient colorimetric detection, by the naked eye, of a hydrophobic protein such as zein in a wide concentration range, covering from $12-3$ $000 \quad$ 1. $\mathrm{g} \mathrm{mL}$

The working principle of the facile colorimetric detection of zein in aqueous ethanol via zein-pyridine-gold interactions is schematically illustrated in Fig. 1. Pyridine-functionalized ScNPs were prepared from copolymers of methyl methacrylate and 4-vinyl pyridine ( $15 \mathrm{~mol} \%$ ) by intrachain folding / collapse using 1,4-diiodobutane as cross-linker (see Supporting Information, SI). As observed for other methyl methacrylate-based copolymers, 17 both the precursors and the pyridine-containing ScNPs were found to be soluble in aqueous ethanol even if actually they are not soluble in pure water or absolute ethanol. It should be noted that to guarantee a complete solubility in aqueous ethanol, the ScNPs were only lightly cross-linked (10 mol \%) so a significant amount of free pyridine moieties remains available in the ScNPs. As schematically illustrated in Fig. 1, addition of 1 eq. of $\mathrm{NaAuCl} 4$ to a transparent solution of pyridine-functionalized ScNPs in ethanol/water (75/25 vol.\%) (Fig. 1a) produces an exchange reaction between I- and $\mathrm{AuCl} 4$ - counter-ions, as well as the formation of $\mathrm{AuCl} 3$-pyridine complexes without colour change in the solution (Fig. 1b). Upon addition of a reducing agent such as hydrazine, a change in colour from transparent to red was observed (Fig. 1c) due to AuNP formation, as confirmed by UVvis spectroscopy and TEM measurements (Fig. 2). The average size of the AuNPs was estimated to be $8.0 \mathrm{~nm}$ by the corrected formula of Haiss et al.18 (eq. 11 in ref. 18), in good agreement to the size observed by TEM $(7.5 \pm 2.1 \mathrm{~nm})$. In the presence of zein, reduction by hydrazine leads to a deep blue solution (Fig. 1d) suggesting the formation of larger AuNPs, as confirmed by TEM showing the presence of AuNPs of ca. $100 \mathrm{~nm}$ (Fig. 2b, right). As illustrated in Fig. 1e, if zein is added to a solution in which AuNPs have been first formed and remain stabilized by pyridine-containing ScNPs, no such a change in colour (from red to blue) takes place. We hypothesize that if zein is present at high concentration in the solution prior to the gold reduction step, it is able to interact so strongly with the pyridine moieties of the ScNPs that AuNP formation occurs mainly in the solution, without the steric stabilizing effect of the pyridinefunctionalized ScNPs. Consequently, the subtle balance of interactions between zein, pyridine and gold ions in aqueous methanol solutions containing pyridinefunctionalized ScNPs paves the way to the simple and fast colorimetric detection of zein. 
It is worth of mention that a significant affinity between zein residues and pyridine has been previously reported based on 13C NMR measurements.19 Consequently, we established a facile and robust procedure for the colorimetric detection of zein in aqueous ethanol by the naked eye. This modified procedure when compared to that followed in Figure 1 was adapted for the detection of zein in real samples (see SI). The procedure is very simple and involves: i) to an aqueous ethanol solution containing zein, adding pyridine-functionalized ScNPs (15 mol\% pyridine) to a final concentration of $0.5 \mathrm{mg} \mathrm{mL}-1$, ii) adding $\mathrm{NaAuCl} 4$ salt (1 eq. with respect to pyridine moieties) and, finally, iii) adding hydrazine ( 1 drop, $25 \mu \mathrm{L}$ ). In the presence of zein, a colour change is observed almost instantaneously when compared to a reference solution without protein. Fig. 3a illustrates the resulting colours of a range of solutions of decreasing concentration of zein, when compared to the control solution. As can be seen, the method allows detecting zein at concentration as low as 12 1 which is a 10-fold increase in detection limit (LOD) with respect to the previously reported colorimetric method based on stained zein spots on chromatography paper. Moreover, the detection time reduces from more than $1 \mathrm{~h}$ of the previous method to a few seconds of this new procedure, involving a simpler work-up. Fig. $3 \mathrm{~b}$ shows the UVvis spectra corresponding to the solutions illustrated in Fig. 3a. Upon increasing zein concentration, a progressive shift in the position of the surface plasmon resonance peak is found (Fig. 3c). A linear response is observed in Fig. $3 \mathrm{c}$ in the zein concentration range from 12 to $100 \mu \mathrm{g} \mathrm{mL-1}$, while a saturation behaviour is observed at very high zein concentration in solution (> $3000 \mu \mathrm{g} \mathrm{mL}-1$ ).

In summary, we have developed a simple, fast, highly sensitive and robust colorimetric detection of zein in aqueous ethanol based on the formation of AuNPs in the presence of pyridine-functionalized ScNPs. The principle of this colorimetric sensor is based on the subtle balance of zein-pyridine-gold interactions, allowing zein detection by the naked eye in a wide concentration range, covering from $12-3000$ $\mu \mathrm{g} \mathrm{mL}-1$. More importantly, the procedure is amenable for extension to detect other relevant prolamine proteins such as $\mathrm{H}$ hordein, the storage protein of barley which is soluble in hot $70 \%$ ethanol.

Financial support from the Projects MAT2012-31088 (MINECO) and T-654-13 (GV) is acknowledged. A. L.-S. is grateful to the University of the Basque Country for his UPV/EHU pre-doctoral grant. 


\section{Notes and references}

1 Zein: Structure, Production, Film Properties and Applications. In: Natural Polymers: Volume 1: Composites. Editors: M. J. John, S. Thomas. RSC publishing, 2012.

2 R. Sridhar, R. Lakshminarayanan, K. Madhaiyan, V. A. Barathi, K. H. C. Lim, S. Ramakrishna, Chem. Soc. Rev., 2015, 44, 790.

3 H. Liang, Q. Huang, B. Zhou, L. He, L. Lin, Y. An, Y. Li, S. Liu, Y. Chen, B. Li, J. Mater. Chem. B, 2015, 3, 3242.

4 U. Shimanovich, G. J. L. Bernardes, T. P. J. Knowles, A. Cavaco-Paulo, Chem. Soc. Rev., 2014, 43, 1361.

5 A. Mahal, P. Khullar, H. Kumar, G. Kaur, N. Singh, M. Jelokhani-Niaraki, M. S. Bakshi, ACS Sustainable Chem. Eng., 2013, 1, 627.

6 S. Podaralla, R. Averineni, M. Alqahtani, O. Perumal, Mol. Pharmaceutics, 2012, 9, 2778.

7 Y. Li, Q. Xia, K. Shi, Q. J. Huang, Phys. Chem. B, 2011, 115, 9695.

8 T. J. Anderson, B. P. Lamsal, Cereal Chem., 2011, 88, 159.

9 A. Esen, Cereal Chem., 1980, 57, 129.

10 M. Gonzalez-Burgos, A. Latorre-Sanchez, J. A. Pomposo, Chem. Soc. Rev., 2015, DOI: 10.1039/C5CS00209E.

11 C. K. Lyon, A. Prasher, A. M. Hanlon, B. T. Tuten, C. A. Tooley, P. G. Frank, E. B. Berda, Polym. Chem., 2015, 6, 181.

12 L. Li, K. Raghupathi, C. Song, P. Prasad, S. Thayumanavan, Chem. Commun., 2014, 50, 13417.

13 O. Altintas, C. Barner-Kowollik, Macromol. Rapid Commun., 2012, 33, 958.

14 L. Dykman, N. Khlebtsov, Chem. Soc. Rev., 2012, 41, 2256.

15 M. A. J. Gillissen, I. K. Voets, E. W. Meijer, A.R. A. Palmans, Polym. Chem., 2012, 3, 3166.

16 J. He, L. Tremblay, S. Lacelle, Y. Zhao, Soft Matter, 2011, 7, 2380.

17 R. Hoogenboom, H. M. L. Thijs, D. Wouters, S. Hoeppener, U. S. Schubert, Soft Matter, 2008, 4, 103.

18 W. Haiss, N. T. K. Thanh, J. Aveyard, D. Ferning, Anal. Chem., 2007, 79, 4215.

M. Augustine, I. C. Baianu, J. Food Sci., 1987, 52, 649. 


\section{FIGURES}

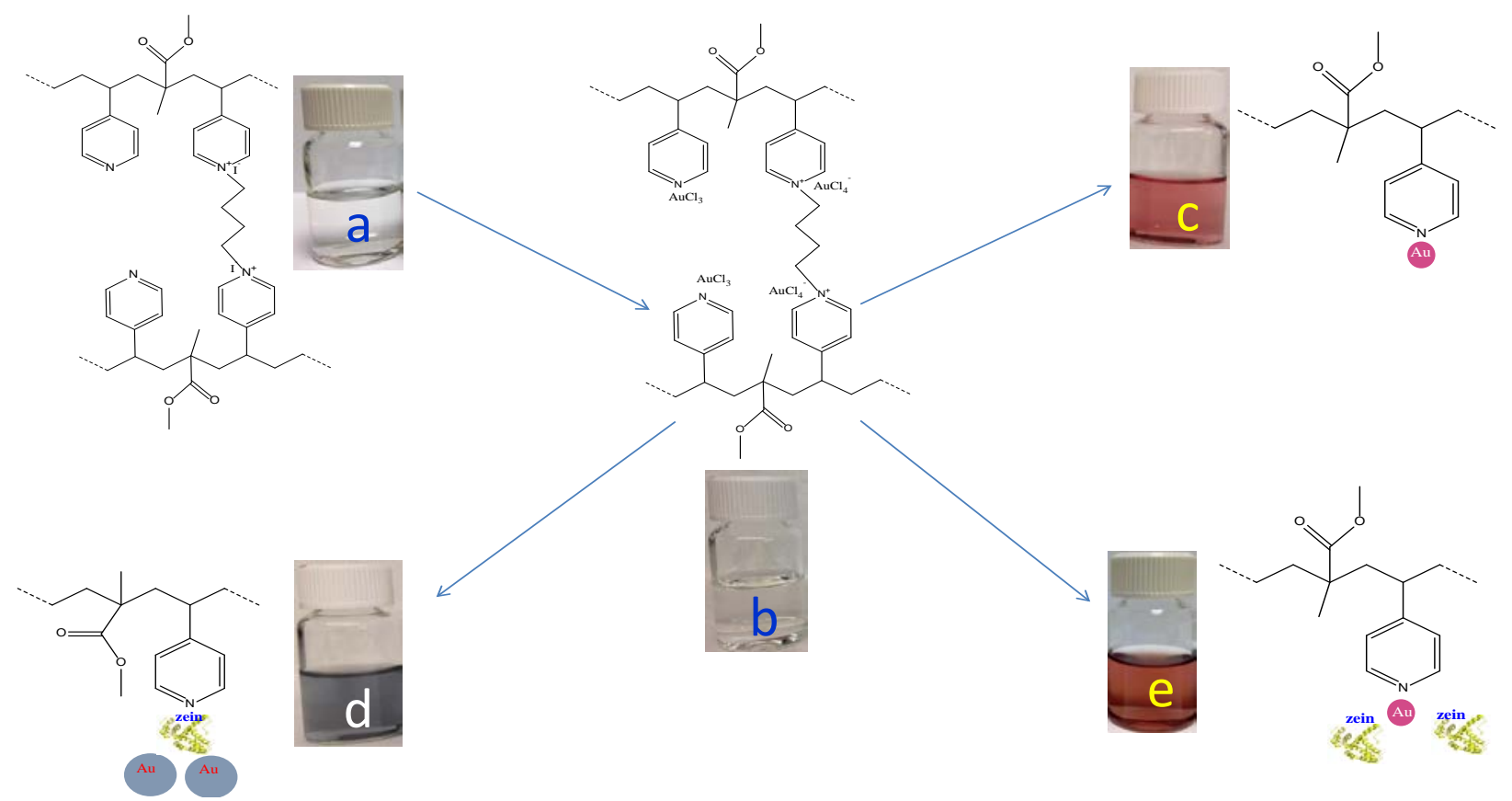

Fig.1. The principle of the colorimetric sensor for zein in aqueous ethanol based on zein-pyridine-gold interactions: In absence of zein, a transparent solution of pyridine-functionalized ScNPs $\left(0.5 \mathrm{mg} \mathrm{mL}^{-1}\right)$ (a) takes a red colour (c) upon AuNP formation by $\mathrm{Au}(\mathrm{III})$ reduction with a drop of hydrazine $(25 \mu \mathrm{L})$. When zein is first added to solution denoted (b) and then a drop of hydrazine, the colour of the solution becomes blue (d). On the contrary, when a drop of hydrazine is first added to (b) and then zein, the colour of the solution becomes red (e). 
A)

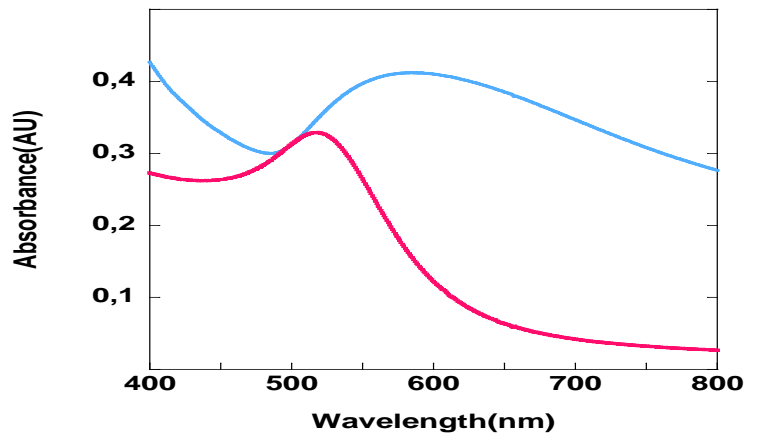

B)

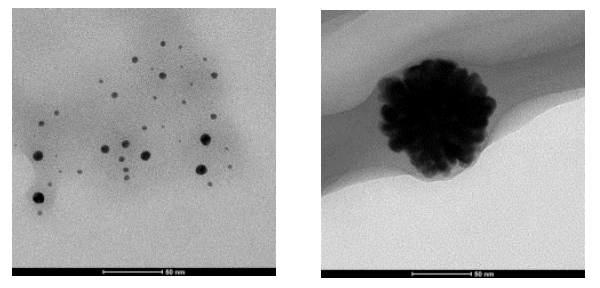

Fig. 2. (a) UV-vis spectra corresponding to the solutions shown in Fig. 1c (in red) and 1d (in blue). (b) TEM images at the same magnification of AuNPs from the solutions shown in Fig. 1c (left) and 1d (right). 

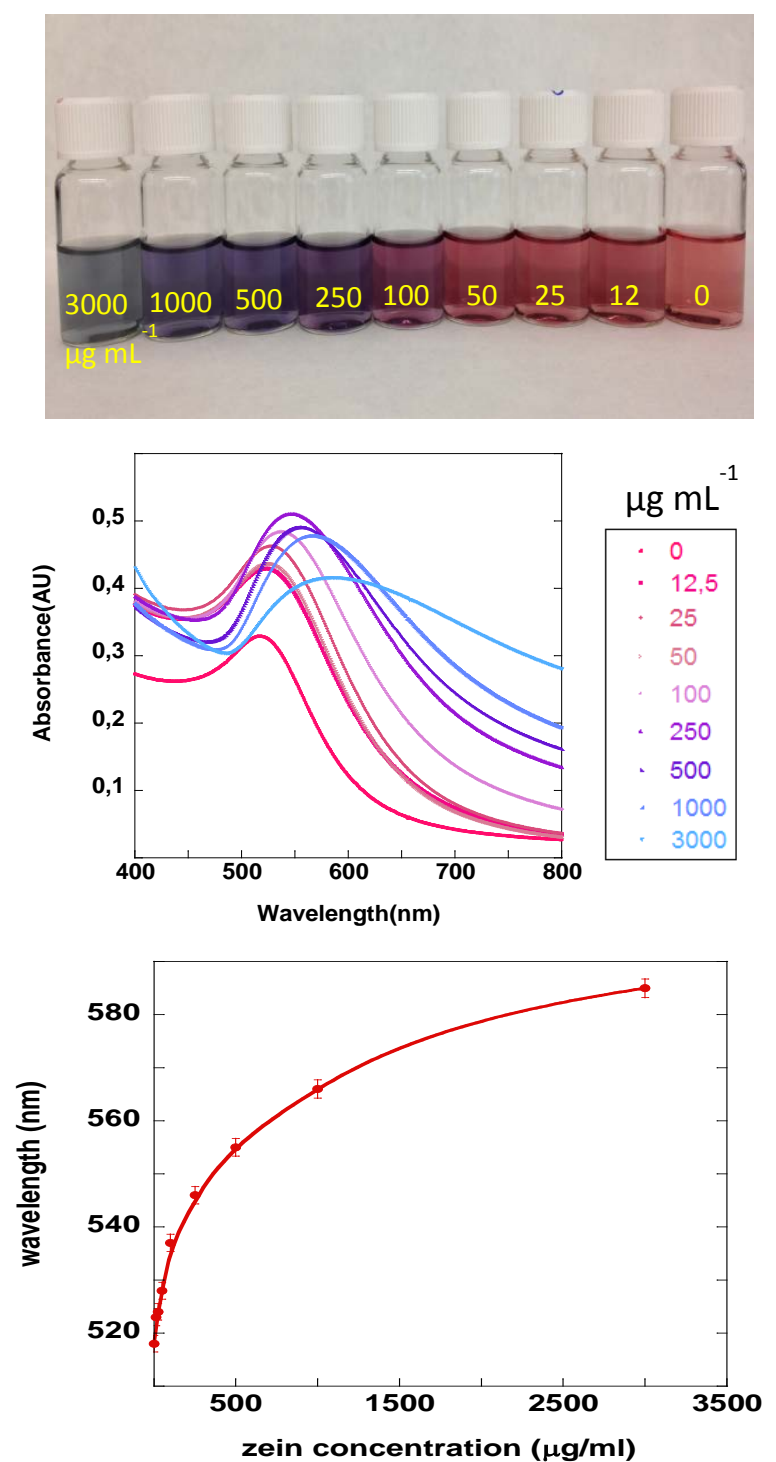

Fig. 3. (a) Photographs of the sensing system in the presence of decreasing concentrations of zein. (b) UV-vis spectra corresponding to the above solutions. (c) Evolution of the position of the maximum of the UV-vis band with zein concentration. 